\title{
Comparative Observation on the Effect of Vancomycin-Loaded Calcium Sulfate on Promoting the Natural Healing of the Docking site of Bone Transport
}

\section{Xuede Guo}

Bozhou City People's Hospital

\section{Guanjun Cui}

Bozhou City People's Hospital

\section{Cishan Wang}

Bozhou City people's Hospital

\section{Hongya Meng}

Bozhou City people's Hospital

\section{Shujie Huang}

Henan orthopedic hospital

Zhuqing Zha ( $\nabla$ zzqingcui2012@163.com )

Henan orthopedic Hospital https://orcid.org/0000-0002-7064-5838

\section{Research article}

Keywords: bone transport, bone defect, calcium sulfate

Posted Date: February 1st, 2021

DOI: https://doi.org/10.21203/rs.3.rs-154466/v1

License: (c) (i) This work is licensed under a Creative Commons Attribution 4.0 International License. Read Full License 


\section{Abstract}

Objectives: Observe the efficacy of vancomycin-loaded calcium sulfateखVCS囚in promoting the natural healing of bone transport's docking site.

Methods: We retrospectively analyzed 48 patients who used bone transfer to treat the tibia's infectious bone defects from 2013 to 2018 . The patients were randomly divided into an experimental group receiving VCS treatment or a control group not receiving VCS treatment. After treatment, the two groups of docking site healing time, skin incarceration rate, natural healing rate, external fixation index, bony results, and functional results were compared.

Results: The experimental group achieved bone healing in the docking site union for an average of $(15.30 \pm 6.32)$ months after the operation. Among them, 25 cases healed spontaneously, two patients healed after bone cleaning and grafting; the average external fixation index was $(2.10 \pm 0.28)$ months/cm; Bone results: excellent in 19 cases, right in 7 patients, low in 1 case, functional derivatives: excellent in 16 points, good in 9 cases, and fair in 2 cases. The control group achieved bone healing at the docking site for an average of $(15.29 \pm 5.36)$ months after the operation. Among them, 11 cases healed spontaneously at the docking site, and 10 cases of non-natural healing ( 6 points of skin incarceration) healed after cleaning and bone grafting; the average external fixation index was $(2.09 \pm 0.25)$ months/cm; Bone results: excellent in 15 cases, right in 5 patients, fair in 1 case, functional products: excellent in 12 points, good in 8 cases, and fitting in 1 case. There was no significant difference in the healing time, bony results, functional results, and external fixation index between the two groups at the docking site ( $P>0.05)$. The experimental group's skin incarceration rate was lower than that of the control group, and the natural healing rate was higher than that of the control group $(P<0.05)$.

Conclusion: In bone removal surgery, the implantation of VCS in the infected bone defect area helps prevent the docking site skin incarceration and promotes the docking site's natural healing.

\section{Introduction}

Bone defects are caused by various reasons, such as trauma, tumor, infection, etc. [1]. The treatment of bone defects has always been a difficult problem in the medical field. The current methods used to treat infectious bone defects mainly include the induced membrane technology pioneered by Masquilet and Ilizarov's distraction osteogenesis [2]. Related studies have found that the llizarov technique has been widely used in tibial bone defects and has gradually become the primary treatment method for infectious bone defects[2]. The llizarov technology (bone handling technology) can generate reliable quality new bone through the principle of distraction osteogenesis and has an excellent clinical effect on segmental bone defects $[3,4]$. However, some cases cannot heal naturally at the docking site. It may be necessary to clean up and graft the bone. A common complication after bone removal is that nonunion at the docking site has attracted more attention [5-8]. Calcium sulfate(CS)has good biocompatibility and degradability; it is a good bone filling material and is often used as a drug carrier to treat infectious bone defects [9]. At present, a combination of antibiotics loaded with CS is commonly used to treat contagious bone defects. There are 
few reports in the literature about the comparative observation of the docking site's natural healing rate with or without VCS implantation during bone transportation. Therefore, we collected tibial infectious bone defects treated by bone transfer in Bozhou People's Hospital and Henan Orthopedic Hospital from January 2013 to January 2018. Groups were grouped according to whether VCS was implanted in the bone defect area after debridement of the infected foci. The natural healing of the docking site was compared and observed.

\section{Materials And Methods General Information}

Regression analysis of patients with the tibia's infectious bone defect was treated in our hospital and cooperative units from January 2013 to January 2018, mainly including closed tibial fracture internal fixation, tibial open fracture, and chronic tibial osteomyelitis. According to the eligibility criteria, all patients were followed up and evaluated(Table 1). All patients included in the study signed an informed consent form, and the ethics committee approved our review of our hospital and our partner unit.

Table 1 Eligibility criteria of the study.

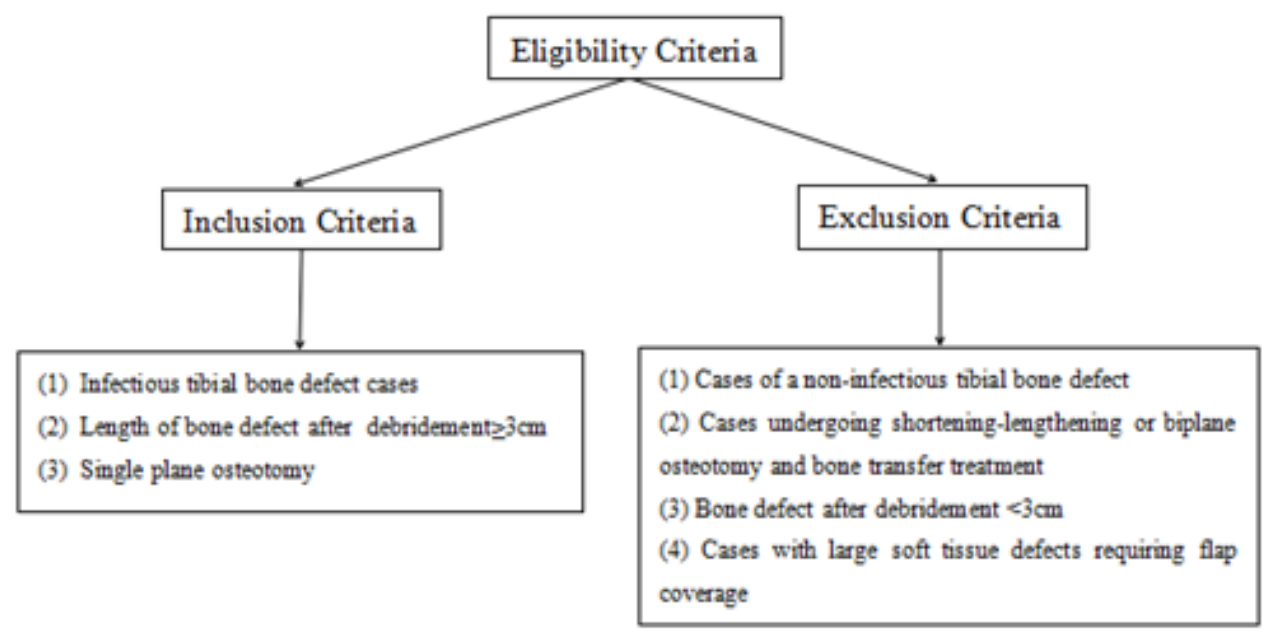

A total of 48 patients meeting the criteria were included in the study. The patients included in the study were divided into two groups: an experimental group and a control group. The experimental group (VCS implantation group) 27 cases: 23 males and four females; age $25-73$ years old, average $(48.81 \pm 11.07)$ years old; The course of the disease was 1 to 86 months, with an average of $(21.00 \pm 16.08)$ months; 17 cases of the infectious bone defect after internal fixation of closed tibial fractures, 8 cases of contagious bone defect of open tibial fractures, 2 cases of chronic blood-borne osteomyelitis of tibia with the bone defect; The length of the bone defect after debridement was $3.5 \mathrm{~cm}$ to $14 \mathrm{~cm}$, with an average of $(7.52 \pm$ 2.34) $\mathrm{cm}$. Control group (no VCS implantation group) 21 cases: 18 males and three females; age 28-66 years old, average (50.29 \pm 9.99$)$ years old; The course of the disease was 1 to 38 months, with an average of (17.67 \pm 10.54$)$ months; 14 cases of an infectious bone defect after internal fixation of closed tibial fractures, 6 cases of contagious bone defect of open tibial fractures, 1 case of tibia chronic hematogenous osteomyelitis with the bone defect; The length of the bone defect after debridement was $3.5 \mathrm{~cm}-13.5 \mathrm{~cm}$, with 
an average of $(7.68 \pm 2.58) \mathrm{cm}$. There was no significant difference in gender, age, cause classification of infectious bone defect, disease course, and length of the bone defect after debridement between the two groups $(P>0.05)$ (Table 2$)$.

Table 2 Comparison of basic preoperative data between EG and CG groups

\begin{tabular}{|c|c|c|c|c|c|c|}
\hline Group & $\begin{array}{l}\text { Cases } \\
\nabla n \rrbracket\end{array}$ & $\begin{array}{l}\text { Gender } \\
\text { (n) }\end{array}$ & $\begin{array}{l}\text { Age } \\
\text { (years) }\end{array}$ & $\begin{array}{l}\text { Etiology classification } \\
\text { of } \\
\text { infectious bone defect } \\
\text { (n) }\end{array}$ & $\begin{array}{l}\text { Course of } \\
\text { Disease } \\
\text { (month) }\end{array}$ & $\begin{array}{l}\text { Bone } \\
\text { defect } \\
\text { length } \\
\text { 『cm } \rrbracket\end{array}$ \\
\hline & & Male/Female & $\mathrm{X} \pm s$ & $\begin{array}{l}\text { (Internal fixation of } \\
\text { fractures/ } \\
\text { open } \\
\text { fractures/osteomyelitis) }\end{array}$ & $\mathrm{X} \pm s$ & $\bar{X} \pm s$ \\
\hline CG & 21 & $23 / 4$ & $50.29 \pm 9.99$ & $14 / 6 / 1$ & $17.67 \pm 10.54$ & $7.68 \pm 2.58$ \\
\hline $\begin{array}{l}x^{2} / t \\
\text { value }\end{array}$ & - & 0.000 & 0.476 & 0.162 & 0.822 & 0.221 \\
\hline $\begin{array}{l}P \\
\text { value }\end{array}$ & - & 1.000 & 0.636 & 0.922 & 0.416 & 0.826 \\
\hline
\end{tabular}

Experment group(EG),Control group(CG)

\section{Surgical Methods}

In the supine position, the patient uses general anesthesia or combined spinal-epidural anesthesia, covers the infected wound and surgical field with a protective film, and fixes it with a unilateral rail-type external frame so that the outer structure is parallel to the mechanical axis of the tibia. The screw is perpendicular to the mechanical axis of the tibia. The upper and lower metaphysis were fixed with three screw screws, and the bone segment to be moved was fixed with two screw screws. If the remaining metaphyseal bone segment is too short or the osteoporosis is too heavy to fix three screw nails. After fixation is lacking, the stability adds 1 to 3 at a particular crossing angle between the metaphysis and the above screw nails. A threaded pin is fixed together with a single-sided rail-type outer frame with a combined external fixing frame to increase stability. Continuous drilling osteotomy was used to perform single-plane osteotomy under the metaphysis periosteum to prepare for bone removal. The metaphyseal incision was sutured and wrapped with a sterile dressing. Then remove the infected bone, dead bone, and hardened bone, and trim the bone stump into parallel cross-sections as much as possible. Finally, the lesions were washed alternately with hydrogen peroxide and normal saline and washed with diluted iodophor.

For internal fixation cases, we first remove the inner focus, thoroughly debride the wound, re-sterilize, drape, and finally perform external fixation and metaphyseal osteotomy. 
In the experimental group, the powdered medical CS and vancomycin hydrochloride were mixed at a mass ratio of 10:1, stirred with the matching diluent, and applied to the mold when it was in a paste state. After it has solidified into a bead shape, it is implanted into the bone defect area after debridement. The bone defect area of the control group was left open.

\section{Postoperative Treatment}

According to preoperative bacterial culture results, sensitive antibiotics were administered intravenously for one week after surgery. Instruct patients to exercise limb function, significantly to strengthen nearby joints' exercise and increase the affected limb's axial stress stimulation through pedaling actions. Bone removal was started one week after surgery, with $1 / 6 \mathrm{~mm}$ each time, 4- 6 times a day. After the operation, take pictures for review monthly and adjust the bone migration speed according to the growth of new bone and the patient's tolerance. After the bone stump meets, slow down the bone moving speed, and continue to slowly move the bone for about two weeks (about 20-30 times in total) to keep the docking site in a state of compression. After the bone stump meeting, the patient was instructed to start walking with crutches and part of the weight-bearing. After the docking site is clinically healed, the affected limb's weight-bearing is gradually increased until the weight is fully loaded. When the patient walks with full weight, and there is no pain in the docking site and new bone, gradually loosen the external fixation frame or reduce the external fixation screws. According to the imaging findings, the outer fixation frame was removed after about three months.

In the following cases, the docking site is cleaned up, and the autogenous iliac bone is grafted: there is skin incarceration between the bone stumps; the bone stumps are conical or axially offset, and the bone contact area is small; After the bone stump reunion, compression fixation was taken for two months to observe the docking site without signs of healing.

\section{Observation Index}

Observe whether the docking site has skin incarceration after surgery, whether the docking site heals naturally and the healing time, whether there is a recurrence of infection or re-fracture, external fixation index, etc. According to Paley's infectious nonunion evaluation criteria [10], observe bony results and functional results.

\section{Statistical analysis}

The SPSS 22.0 statistical software was used for statistical analysis of the two groups of observation indicators. The measurement data is expressed as \pm . Comparing age, disease course, bone defect length, and external fixation index between groups was performed using two independent samples $t$ test. Counting data is expressed in frequency. Comparing the two groups of patients' gender, etiology classification, Skin incarceration rate on docking site, docking site natural healing rate, bony results, and functional results were 
compared by $\chi^{2}$ test. The test level $a$ value was taken as two-sided $0.05, P<0.05$ considered the difference statistically significant.

\section{Results}

All patients were followed up for $26-40$ months, with an average of $(31.18 \pm 3.38)$ months, and no secondary vascular and nerve damage occurred. The imaging of VCS beads on X-ray film gradually fades with time and disappears after 5 to 8 weeks( Fig. 1).

The experimental group achieved the docking site's bony union 7 to 37 months after the operation, with an average of $(15.30 \pm 6.32)$ months. Twenty-five cases of the docking site healed spontaneously, and two patients were recovered after the docking site was cleaned and grafted. One point of re-fracture occurred (located in the new bone segment and healed by plaster fixation), one case of clubfoot deformity, no skin incarceration occurred, no infection recurrence; the average external fixation index was ( $2.10 \pm 0.28)$ months/cm. According to Paley's evaluation criteria, the bony results were excellent in 19 cases, right in 7 patients, and low in 1 case. The functional results were excellent in 16 cases, right in 9 points, and fair in 2 cases. The two patients with a docking site that did not heal naturally had poor compliance. One patient refused to continue bone removal due to a needle infection when the bone stump approached the meeting. After removing the external fixator, debridement of the needle path, cleaning the docking site, autogenous bone grafting, and plaster fixation, the needle path infection was cured. The docking site healed. Another patient had a mild mental disorder and could not perform bone removal and limb function exercises prescribed by the doctor. The bone stump reunion was severely delayed, the docking site was conical, and the docking site was cleared and healed after autogenous bone grafting, leaving clubfoot deformity.

The control group achieved bony union from 7 months to 24 months after the operation, with an average of $(15.29 \pm 5.36)$ months of the bone union. Among them, 11 cases were spontaneously healed, and ten patients were not cured naturally (6 Cases with skin incarceration) healed after debridement and bone grafting at the docking site; 1 case of clubfoot deformity, 1 case of infection recurrence (cured after debridement), no re-fracture cases; average external fixation index was $(2.09 \pm 0.25)$ months / cm; According to Paley's evaluation criteria, the performance of bony results: excellent in 15 cases, right in 5 patients, and fair in 1 case. The functional results were as follows: excellent in 12 patients, good in 8 patients, and fitting in 1 case. There were no statistically significant differences in the healing time, bony results, functional results, and external fixation index between the two groups $(P>0.05)$. The experimental group's skin incarceration rate was lower than that of the control group, and the natural healing rate was higher than that of the control group $(P<0.05)$ (Table 3$)$.

Table 3 Comparative analysis of the results of observation indicators in EG and CG groups 


\begin{tabular}{|c|c|c|c|c|c|c|c|}
\hline Group & $\begin{array}{l}\text { Cases } \\
\text { \n囚 }\end{array}$ & $\begin{array}{l}\text { Skin } \\
\text { incarceration } \\
\text { \n『(Yes/no) }\end{array}$ & $\begin{array}{l}\text { Natural } \\
\text { healing } \\
\text { nn } \\
\text { (Yes } \\
\text { /no) }\end{array}$ & $\begin{array}{l}\text { Natural } \\
\text { healing } \\
\text { time } \\
\text { 『month } \rrbracket\end{array}$ & 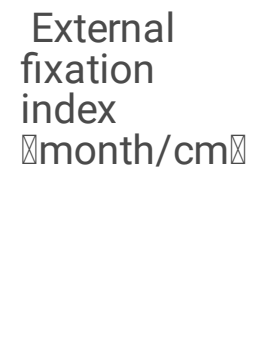 & $\begin{array}{l}\text { Bony } \\
\text { results } \rrbracket n \rrbracket \\
\text { (excellent } \\
\text { /good } \\
\text { /moderate } \\
\text { /poor) }\end{array}$ & $\begin{array}{l}\text { Functional } \\
\text { result } \ \text { n } \varnothing \\
\text { (excellent } \\
\text { /good } \\
\text { /moderate } \\
\text { /poor) }\end{array}$ \\
\hline EG & 27 & $27 / 0$ & $25 / 2$ & $15.30 \pm 6.32$ & $2.10 \pm 0.28$ & $19 / 7 / 0 / 1$ & $16 / 9 / 2 / 0$ \\
\hline CG & 21 & $15 / 6$ & $11 / 10$ & $15.29 \pm 5.36$ & $2.09 \pm 0.25$ & $15 / 5 / 1 / 0$ & $12 / 8 / 1 / 0$ \\
\hline $\begin{array}{l}\mathrm{t} \\
\text { value }\end{array}$ & - & 11.043 & 8.155 & 0.006 & 0.185 & 2.087 & 0.001 \\
\hline $\begin{array}{l}\mathrm{p} \\
\text { value }\end{array}$ & - & 0.004 & 0.002 & 0.006 & 0.854 & 0.555 & 0.977 \\
\hline
\end{tabular}

Experment group(EG),Control group(CG)

\section{Discussion}

Infectious bone defects are currently commonly treated with bone transport technology to achieve good results. However, due to the long period required for bone transport, the osteogenic activity decreases when the bone stump meets, and the skin and soft tissue between the bone stumps are embedded. Suddenly and other factors, some cases cannot heal naturally. Some surgeons advocate routinely cleaning and grafting the docking site after the bone stump meets to promote the docking site healing and shorten the outer frame's wearing time [11]. However, most infectious bone defect cases have undergone multiple operations, local scar formation, and low soft tissue coverage. The second operation increases surgical trauma and medical expenses and risks re-infection or skin and soft tissue necrosis. The application of axial pressure and the "accordion" technique can stimulate bone formation to a certain extent [12-14], promoting bone healing. However, it should be noted that for cases of skin incarceration between the bone stumps, it is not advisable to apply stress measures such as axial compression on the docking site or "accordion" techniques. Otherwise, the skin squeezed between the bone stumps may become necrotic and even lead to bone exposure.

After bone removal, on the one hand, due to the bone segment's ouster, the skin and soft tissue of the bone defect area lack support and sink, and local fibrous scar tissue is generated. On the other hand, with the sliding of the bone segments, the soft tissue is also pushed forward to a certain extent and accumulates between the bone stumps. All of the above factors cause skin and soft tissue incarceration between the bone stumps. In the clinical application of bone transfer, the bone stump has a higher probability of skin and soft tissue incarceration[7, 8]. The skin and soft tissue incarceration make it difficult for the bone stump to achieve accurate bone contact, which adversely affects the docking site healing. In bone removal surgery, vancomycin-loaded calcium sulfate is used to fill the cavity formed after bone segment resection, which can support the skin and soft tissues, and prevent the skin and soft tissues from sinking and the formation of fibrous scar tissue between the bone stumps within a certain period, thereby avoiding the skin and soft 
tissues are incarcerated between the bone stumps. In the process of bone removal, as the medical calcium sulfate is gradually absorbed, the bone stump slowly approaches until it meets the teacher, achieving direct bone contact with the bone stump, which is beneficial to the natural healing of the docking site.

Our follow-up found that VCS development time on X-ray film is 5 to 8 weeks after surgery. Theoretically, according to the bone moving speed of $1 \mathrm{~mm} / \mathrm{d}$ starting one week after surgery, a $5 \mathrm{~cm}$ bone defect can achieve bone stump reunion at about eight weeks after surgery. During this period,VCS can help prevent bone stumps in the skin and soft tissue incarceration. In implanting VCS, even if the bone defect is more significant than $5 \mathrm{~cm}$, there is no skin and soft tissue incarceration between the bone stumps. We speculate that the possible reason is that after the VCS is completely degraded, the surrounding soft tissues will not wholly occupy the potential channels formed by the VCS degradation in the short term.

Besides, we found a specific positive correlation between the retention time of VCS beads and the beads' size. The larger the dots, the longer the retention time. To slow down the degradation of VCS beads, it is essential for bone defects. For cases with longer segments, larger VCS beads should be implanted. We make VCS into beads instead of rods. The advantage is that as the bone moves, the VCS can be pushed forward by the bone stump without hindering the bone stump's advancement. Although the implantation of VCS can better promote the docking site's natural healing, it is not a single factor that affects the docking site's natural recovery. Therefore, it is necessary to prevent the influence of other unfavorable factors simultaneously, such as avoiding the axial deviation of the bone segment, applying docking site compression and stress stimulation. These adverse factors on the natural healing of docking site treatment by bone handling technology need to be further studied.

\section{Conclusion}

Through the comparative analysis of the two groups of observation indicators, we can conclude that the implantation of VCS in the infected bone defect area helps prevent the docking site's skin incarceration and promote the natural healing of the docking site. However, the natural healing of a docking site is affected by many factors, which requires further research. Besides, this study is a retrospective clinical observation, and a rigorous large-sample prospective controlled study is still needed.

\section{Abbreviations}

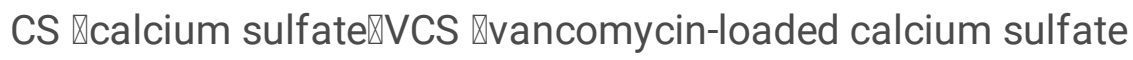

\section{Declarations}

\section{Acknowledgements}

None.

\section{Authors' contributions}


XDG and ZGZ proposed research ideas and designed the research. GJC and XDG wrote manuscripts, statistical analysis and drawing of pictures. HYM, CSW and SJH contribute to data collection and data quality control. All authors have read the manuscript and agreed to the final version of the manuscript.

\section{Funding}

Scientific Research Project of Bozhou People's Hospital (By201933); Project name: Application research of Ilizarov technology. Three New Projects of Bozhou People's Hospital (2016ZDB-006); Project name: Clinical application research of bone handling technology.

\section{Availability of data and materials}

The data and materials in this article can be obtained by sending an email to the corresponding author as needed.

\section{Ethics approval and consent to participate}

The research plan has been approved by the Medical Ethics Committee of Bozhou People's Hospital and Henan Orthopedic Hospital, and all participants have signed written informed consent.

\section{Consent for publication}

Not applicable.

\section{Competing interests}

No potential conflict of interest was reported by the authors.

\section{References}

[1] Zhu W, Wang D, Xiong J, et al. Study on clinical application of nano-hydroxyapatite bone in bone defect repair. Artif Cells Nanomed Biotechnol. 2015;43(6):361-365.

[2] Aktuglu K, Erol K, Vahabi A. Ilizarov bone transport and treatment of critical-sized tibial bone defects: a narrative review. J Orthop Traumatol. 2019;20(1):22.

[3] Wu YW,Yin QD,Rui YJ,et al.llizarov technique:bone transport versus bone shortening-lengthening for tibial bone and soft-tissue defects[J].J Orthop Sci,2018,23(2):34I-345.

[4] Giannoudis PV.Treatment of bone defects:Bone transport or the induced membrane technique? [J].Injury,2016,47(2):291-292区

[5] Antony D, Jose R.llizarov ring fixator in the management of infected nonunion of tibia-A clinical study[J].Evolution Med.Dent.2016,5(92):6835-6841 
[6] Bukva B, Vrgoc G, Rakovac l, et a1.Complications in leg lengthening using an Ilizarov external fixator and intramedullary alignment in children囚comparative study during a fourteen-year period[J].Injury,2015,46 Suppl 6खS48-51凹

[7] Liu YY, Shen LF, Zhang C, et al. Causes and strategies for postoperative bone nonunion after bone transport of lower limb bone of chronic osteomyelitis with bone defect[J].China J Orthop Trauma,2018,31(6):556-561.

[8] Shen J, Qiao L, Zhang SM, et al. Analysis of end complications of bone transport technique for defects in long bones of lower extremity[J].Chinese Journal of BoneandJointInjury,2017,32(1):49-52.

[9] Kutkut A, Andreana S. Medical-grade calcium sulfate hemihydrate in clinical implant dentistry: a review. J Long Term Eff Med Implants. 2010;20(4):295-301.

[10] Paley D,Catagni MA,Argnani F,et al.llizarov treatment of tibial nonunions with bone loss[J].Clin Orthop Relat Res,1989(241):146-165.

[11] Hu JZ, Shi ZY, Yang CZ, et al. Clinical study of bone transport combined with bone graft and internal fixation at the docking site in the treatment of large segmental bone defect in lower limb[J].Chin $\mathrm{J}$ Orthop,2018,38(5):280-287.

[12] Karargyris O, Polyzois VD, Karabinas P, et al. Papineau debridement, llizarov bone transport, and negative-pressure wound closure for septic bone defects of the tibia.Eur J Orthop SurgTraumatol.2014,24(6):1013-1017.

[13] Xu J,Jia YC,Kang QL,et a1.Management of hypertrophic nonunion with failure of internal fixation by distraction osteogenesis[J].Injury,2015,46(10):2030-2035.

[14] Zhang Q,Zhang W,Zhang Z,et a1.Accordion technique combined with minimally invasive percutaneous decortication for the treatment of bone non-union[J].Injury,2017,48(10:2270-2275.

\section{Figures}



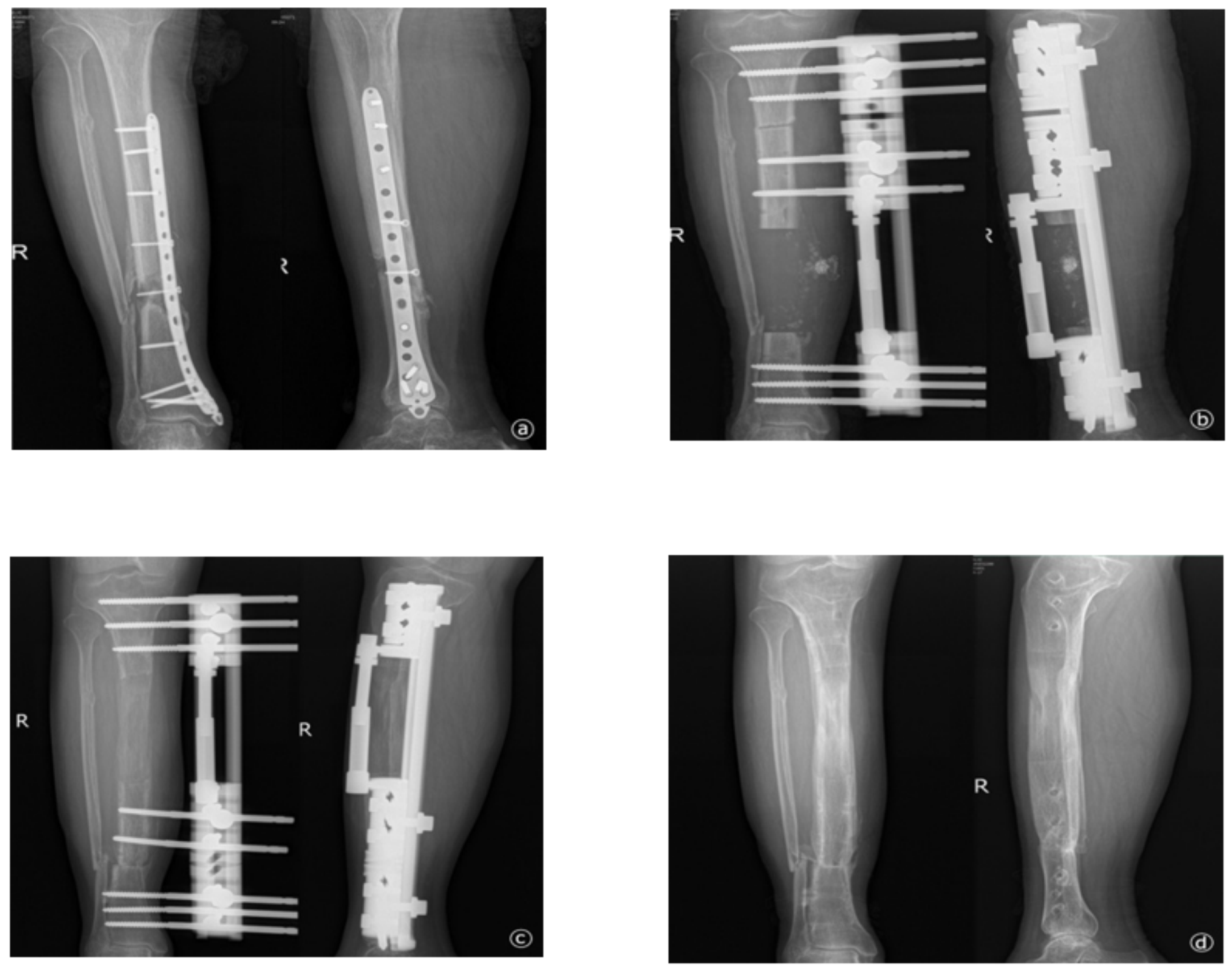

Figure 1

A 52-year-old man with an infectious bone defect after internal fixation of a right tibial fracture underwent bone removal and vancomycin-loaded calcium sulfate implantation. (a) X-ray before operation showed an infectious bone defect of the tibia. (b) Postoperative X-ray film showed vancomycin-loaded calcium sulfate imaging in the bone defect area. (c) X-ray film shows natural healing of the opposite ends in November after the operation, and the screws at both ends are slightly bent due to compression. (d) The outer frame was removed 14 months after the process. X-ray film showed bone mineralization and bone healing. 